\title{
Estrategia individual y poblacional en las intervenciones frente a la epidemia del tabaquismo
}

\section{Individual and population-based strategies for tobacco control}

\author{
Jose M. MARTínez-SÁNCHEZ

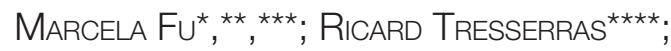

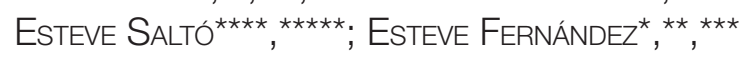

Unitat de Control del Tabaquisme, Programa de Prevenció i Control del Càncer, Institut Català d'Oncologia - ICO, L'Hospitalet de Llobregat.

** Grup de Prevenció i Control del Càncer, Institut d'Investigació Biomèdica de Bellvitge - IDIBELL, L'Hospitalet de Llobregat.

*** Departament de Ciències Clíniques, Facultat de Medicina, Campus de

Bellvitge, Universitat de Barcelona, L'Hospitalet de Llobregat.

**** Departament de Salut, Generalitat de Catalunya.

${ }^{* \star \star \star \star}$ Departament de Salut Pública, Univesitat de Barcelona, Barcelona.

Enviar correspondencia a:

Jose M. Martínez Sánchez

Unitat de Control del Tabaquisme.

Institut Català d'Oncologia

Av Gran Via de L'Hospitalet 199-203

08908 L'Hospitalet de Llobregat (Barcelona)

E-mail: jmmartinez@iconcologia.net

\section{RESUMEN}

La estrategia poblacional de las intervenciones frente a los factores de riesgo se dio a conocer por los trabajos de Geoffrey Rose en el campo de la salud pública y la medicina preventiva de las enfermedades cardiovasculares a finales del siglo XX. En la actualidad, uno de los factores de riesgo con mayor impacto sobre la salud es el tabaquismo. El objetivo de este trabajo es describir y aplicar las estrategias de la medicina preventiva propuesta por Geoffrey Rose en el control de la epidemia del tabaquismo. Para ilustrarlo hemos utilizado los datos de concentración de cotinina en saliva de una muestra representativa de la población adulta de la ciudad de Barcelona. En conclusión, las políticas públicas de control de tabaquismo en todo el mundo deberian tener en consideración la utilidad de la estrategia poblacional para el abordaje de este importante problema de salud, sin menospreciar intervenciones seleccionadas dirigidas a colectivos de "alto riesgo" que sean de probada eficacia.

Palabras clave: control del tabaquismo, estrategia poblacional, estrategia de alto riesgo, cotinina

\section{ABSTRACT}

The population strategy for intervention on risk factors became known through the work of Geoffrey Rose in the field of public health and preventive medicine in relation to cardiovascular diseases in the late twentieth century. Currently, one of the risk factors with the most impact on health is the smoking epidemic. The aim of this study is to describe and put into practice the preventive medicine strategies proposed by Geoffrey Rose in the control of this smoking epidemic. To illustrate the situation, we have used the data on salivary cotinine concentration from a representative sample of the adult population in the city of Barcelona. In conclusion, public tobacco control policies worldwide should take into account the utility of the population strategy for addressing this important health issue, without ignoring "high risk" interventions of proven effectiveness.

Key words: tobacco control, population strategy, high-risk strategy, cotinine 


\section{Introducción}

$\mathrm{L}^{2}$ a estrategia poblacional frente a los factores de riesgo de enfermedades fue ampliamente descrita en los trabajos de Geoffrey Rose en el campo de la medicina preventiva y salud pública de las enfermedades cardiovasculares a finales del siglo $X X^{1,2}$. A partir de preguntas tales como: ¿por qué este paciente tiene esta enfermedad en este momento? ¿qué ocurrió para que esta persona enfermara? y ¿podía haber sido prevenido este caso?, Rose planteó dos tipos distintos de estrategias para hacer frente a los factores de riesgos: la estrategia individual o de "alto riesgo" y la estrategia poblacional. La estrategia individual tiene como objetivo identificar a los individuos con más riesgo de enfermar, focalizando las intervenciones sanitarias en este grupo específico de la población ${ }^{3}$. Por otra parte, la estrategia poblacional tiene como objetivo cambiar la distribución poblacional del factor de riesgo mediante intervenciones dirigidas a toda la población, incluyendo a los individuos de alto y bajo riesgo ${ }^{3}$. Ambas estrategias han sido debatidas ampliamente y tienen sus ventajas e inconvenientes, así como defensores ${ }^{4}$ y detractores $^{5}$. Rose defiende las ventajas de utilizar una estrategia poblacional sobre factores de riesgo tales como la hipertensión arterial, la hipercolesterolemia o el consumo de sal o alcohol ${ }^{1}$. Sin embargo, el tabaquismo, a pesar de ser la principal causas de enfermedad y muerte evitable de los paises desarrollados ${ }^{6-8}$ y uno de los factores de riesgo que tiene mayor impacto sobre la salud, ha sido escasamente conceptualizado desde esta perspectiva9 ${ }^{9}$.

El consumo de tabaco y la exposición al humo ambiental del tabaco (HAT) aumentan el riesgo de padecer enfermedades crónicas como el cáncer de pulmón y enfermedades cardiovasculares y respiratorias ${ }^{6-8}$. En España, se atribuyen casi 54.000 defunciones anuales al tabaquismo activo $0^{10}$ y hasta 3.200 a la exposición pasiva al HAT ${ }^{11}$. Considerando la magnitud de la carga de enfermedad producida por el tabaquismo puede ser de relevante aplicar y estudiar las estrategias propuestas por Rose en el control de esta epidemia.

En términos generales, la epidemia del tabaquismo (consumo del tabaco y exposición pasiva) puede ser monitorizada a partir de métodos indirectos y métodos directos de medición. Entre los métodos indirectos las encuestas (o estudios transversales) han sido el principal recurso y cuantifican subjetivamente el consumo de tabaco y la exposición al HAT. Para cuantificar el consumo de tabaco y la exposición pasiva de una forma objetiva es necesario el uso de un marcador ambiental o biológico (biomarcadores). Entre los biomarcadores individuales, la cotinina ha sido el más utilizado en la literatura científica.

La cotinina es un marcador biológico específico del tabaco, es fácilmente detectable en los fluidos orgánicos y mantiene una razón constante con otros productos del tabaco. La concentración de cotinina en saliva ha sido utilizada para describir algunas característica del consumo de tabaco ${ }^{12}$, estudiar la exposición al HAT en población general ${ }^{13,14}$ y evaluar el impacto de medidas sanitarias frente al tabaquismo en distintas poblaciones ${ }^{13,15-17}$.
La distribución de la concentración de cotinina en la población general ha sido descrita en diversas poblaciones ${ }^{18,}$ ${ }^{19}$, incluyendo la española ${ }^{14,20}$. Todos estos estudios describen la distribución poblacional de la concentración de cotinina como una distribución bimodal donde se diferencia claramente la población fumadora de la no fumadora. Conocer esta distribución en la población general puede ser de gran ayuda para adoptar medidas preventivas y de intervención en el control del tabaquismo.

El objetivo de este trabajo es describir las estrategias individual y poblacional planteadas por Geoffrey Rose en las medidas de control del tabaquismo ilustrándolas con datos de la concentración de cotinina en saliva en una muestra representativa de la población adulta.

\section{Medidas de control del tabaquismo}

En el año 2003 se aprobó el Convenio Marco de la Organización Mundial de la Salud (OMS) para el Control del Tabaco (CMCT) ${ }^{21}$. El CMCT se elaboró en respuesta a la globalización de la epidemia del tabaquismo y reafirma el derecho de todas las personas a gozar del grado máximo de salud que se pueda lograr. Hasta la fecha (mayo 2011), todos los países de la Unión Europea, excepto la República Checa, han ratificado el CMCT. España lo firmó el 16 de junio de 2003 y lo ratificó el 11 de enero de $2005^{22}$.

Después de que el CMCT fuera adoptado por los países miembros de la OMS, el Banco Mundial describió las seis medidas de intervención más costo-eficaces para reducir el impacto del tabaquismo en la salud de la población en base a la literatura científica ${ }^{23}$. Las seis medidas descritas por el Banco Mundial fueron: aumento de los impuestos sobre los cigarrillos y otros productos del tabaco; creación de espacios libres de humo de tabaco en lugares públicos y centros de trabajo (escuelas, centros sanitarios, transportes, cines, restaurantes, etc.); ampliación de las prohibiciones de la publicidad y la promoción de todos los productos, logotipos y marcas de tabaco; mejoras en la información al consumidor sobre los efectos del tabaco; etiquetado de advertencias sanitarias directas y grandes en las cajetillas de cigarrillos y en otros productos del tabaco; y ayuda para que los fumadores puedan dejar de fumar. Estas medidas tienen efectos tanto sobre la salud de los fumadores como de los potenciales fumadores y no fumadores. La evidencia científica indica que los mejores resultados se logran cuando se implementan todas estas medidas de forma integral y conjunta ${ }^{24}$, siendo el aumento de precios del tabaco la manera más eficaz de reducir la prevalencia de consumo de tabaco, especialmente en jóvenes y en grupos sociales con bajos ingresos ${ }^{23}$.

\section{Estrategia individual frente al tabaquismo}

Una visión individual o de "alto riesgo" del problema de salud del tabaquismo pondría el acento en todas las medidas sanitarias de control del tabaquismo que tuvieran como población diana a las personas con mayor riesgo individual. Las personas fumadoras, independientemente del número de 
cigarrillos fumados y su dependencia a la nicotina, son las que tienen mayor riesgo individual asociado al tabaquismo. Por este motivo, las intervenciones de alto riesgo estarian dirigidas exclusivamente a las personas fumadoras.

Una forma de ilustrar el riesgo individual de ser fumador en la población general sería mediante el uso de un biomarcador que cuantifique de manera continua y objetiva los niveles de exposición y riesgos individuales en la población general. En este sentido, la concentración de cotinina en saliva, aunque tiene una corta vida media y puede variar dependiendo del día de recogida, puede discriminar a partir de un determinado punto de corte a las personas que fuman de las que no fuman ${ }^{18,20}$. Además, la concentración de cotinina proporciona información sobre el riesgo individual de desarrollar enfermedad de las personas fumadoras ${ }^{25,26}$. La asunción, basada en las evidencias disponibles ${ }^{7,25}$, es que existe un mayor riesgo individual de desarrollar una enfermedad relacionada con el tabaco cuanto mayor es la concentración de cotinina.

En la figura 1 (panel A) se muestra la distribución poblacional de la concentración de cotinina en saliva en una muestra representativa de la población adulta de la ciudad de Barcelona ${ }^{14}$ (>16 años). La población diana de las intervenciones de "alto riesgo" serían las personas fumadoras. El punto de corte de la concentración de cotinina que discrimina la persona fumadora de la no fumadora en la población adulta española es de $10 \mathrm{ng} / \mathrm{ml}^{20}$. Las concentraciones de cotinina en suero (muy similares a las determinadas en saliva) superiores a $15 \mathrm{ng} / \mathrm{ml}$ se asocian positivamente con desarrollar un cáncer de pulmón ${ }^{25,26}$ siendo las concentraciones de cotinina más elevadas las que tienen mayor asociación con un cáncer de pulmón. En la figura 1 (panel B) el punto de corte que discrimina entre fumadores y no fumadores (10 $\mathrm{ng} / \mathrm{ml}$ ) permite identificar a la población diana de esta posible estrategia de "alto riesgo". Aunque el riesgo de desarrollar una enfermedad asociada al tabaquismo aumenta con el nivel de cotinina, la factibilidad de cuantificar este metabolito de la nicotina en la práctica clínica es limitada. Además el consejo médico para dejar de fumar se realiza de manera sistemática a todos los fumadores independientemente de sus niveles de cotinina. En este sentido, se podría considerar población de "alto riesgo" a todos los fumadores independientemente de sus niveles de cotinina y de su consumo actual de cigarrillos. De las medidas descritas por el Banco Mundial, las encaminadas al abandono del tabaquismo, como los tratamientos para la deshabituación y el consejo médico sistematizado en la consulta del profesional sanitario serian las estrategias que podemos considerar de "alto riesgo". Asimismo, el aumento del precio del tabaco y la mejorara de la información de los efectos perjudiciales para la salud de los fumadores mediante el etiquetado de las cajetillas con imágenes impactantes sobre los efectos del tabaco también pueden considerarse intervenciones de alto riesgo. El resultado esperado y deseable de estas intervenciones de "alto riesgo" sería cierta reducción del número de fumadores y del número de cigarrillos fumados con una posible reducción en la concentración de cotinina en esta población diana. Esta reducción de los niveles de cotinina de la población diana (personas fumadoras) se traduce en una ganancia en salud en términos de reducción de la morbilidad y mortalidad de las enfermedades asociadas al consumo de tabaco como el cáncer de pulmón y el infarto de miocardio.

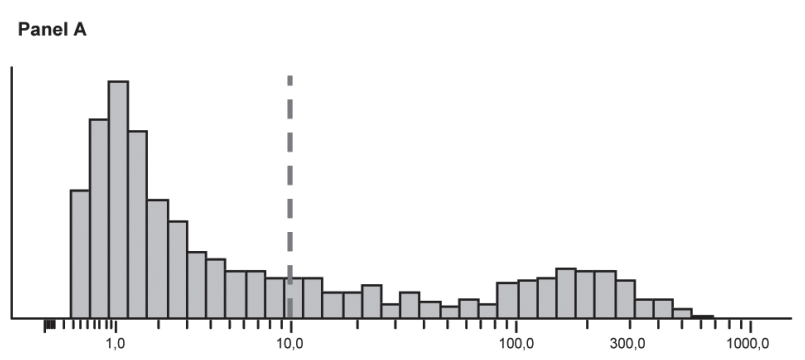

Panel B

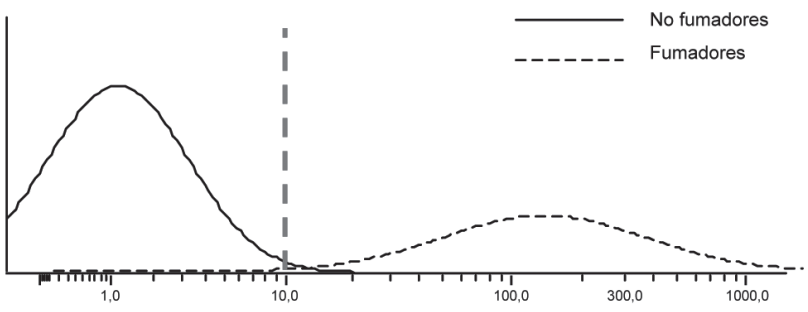

Panel C

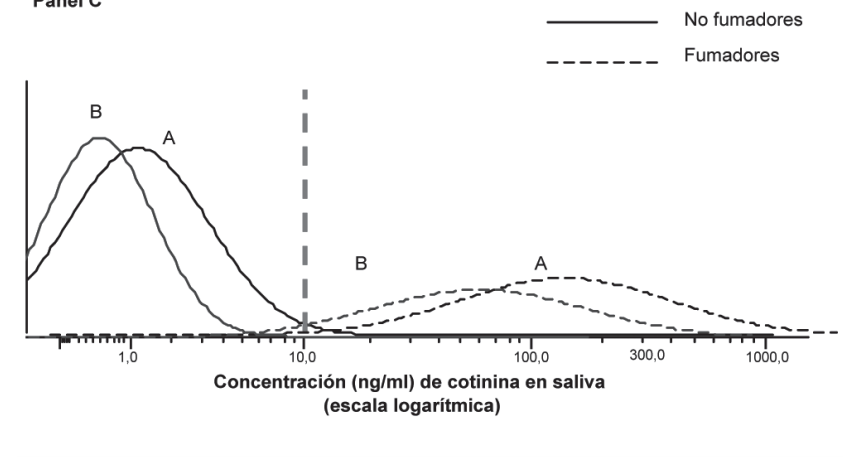

Elaboración propia a partir de los datos del estudio de Martinez-Sánchez et al. ${ }^{14}$

Panel A: Distribución observada en la población general de Barcelona.

Panel B: llustración de la estrategia de alto riesgo.

Panel C: llustración de la estrategia poblacional. Curva A: Distribución observada; curva B: escenario ficticio con el desplazamiento de la distribución debido a la estrategia poblacional. La linea vertical indica el punto de corte considerado a partir del cual se discrimina la población fumadora (de alto riesgo) de la no fumadora (con concentración de cotinina inferiores a $10 \mathrm{ng} / \mathrm{ml}^{20}$.

Figura 1.- Distribución de la concentración de cotinina en saliva en la población adulta ( $>16$ años) no fumadora y fumadora de la ciudad de Barcelona (2004-2005). Ilustración de la estrategia de "alto riesgo" y poblacional.

\section{Estrategia poblacional frente al tabaquismo}

El objetivo principal de la visión poblacional del problema de salud de la epidemia del tabaquismo sería cambiar la distribución poblacional de este factor de riesgo. En este escenario, las medidas de control del tabaquismo irian dirigidas tanto a personas fumadoras como no fumadoras, independientemente de su nivel de riesgo individual. 


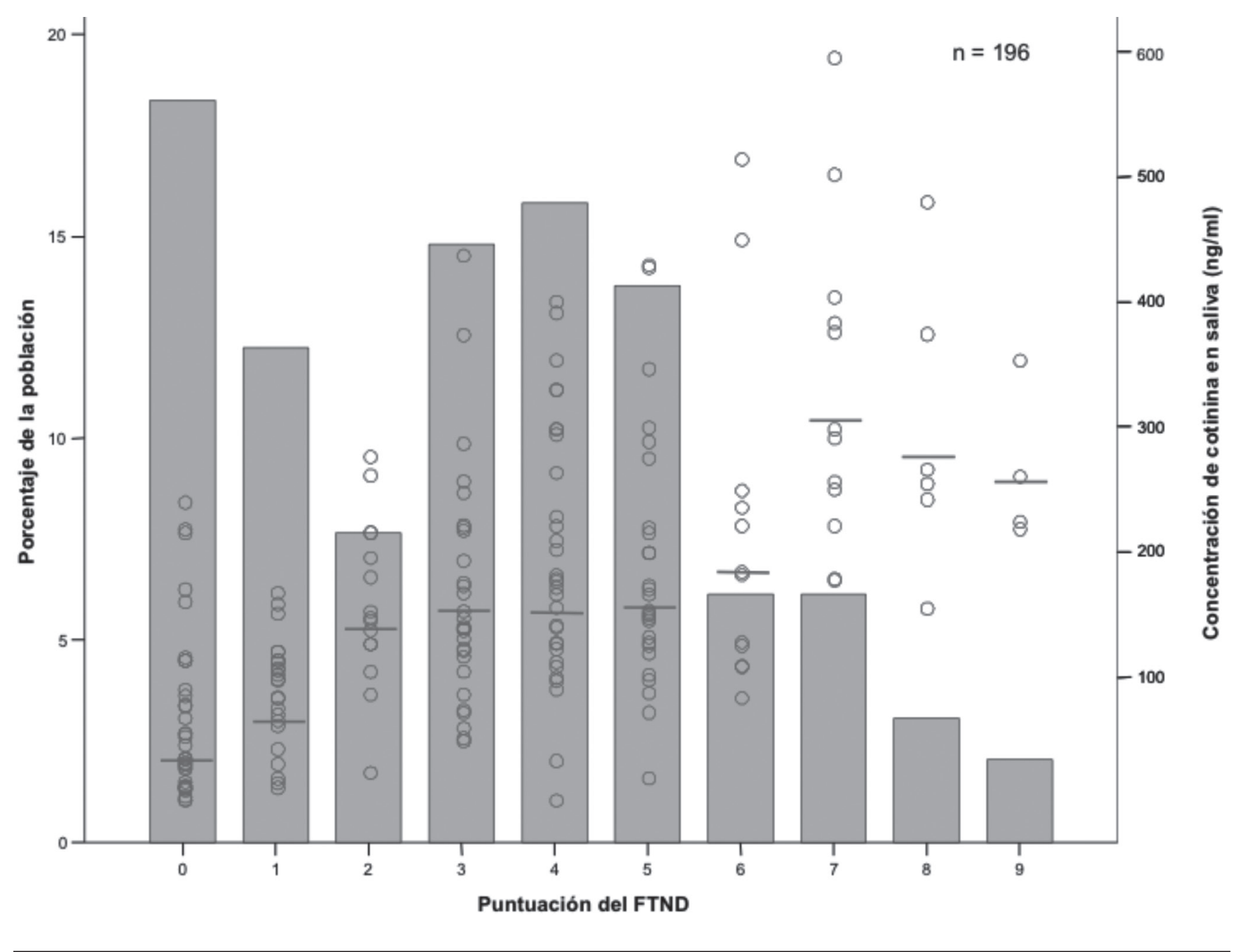

Elaboración propia a partir de los datos del estudio de Fu et al.12

La linea horizontal representa la media de la concentración de cotinina en saliva en cada una de las categorias de la puntuación del test de Fagerström (FTND).

Figura 2.- Distribución poblacional (barras) de una muestra de fumadores diarios de la ciudad de Barcelona según su puntuación en el test de Fagerström y la concentración de cotinina en saliva individual (puntos)

Para ilustrar la estrategia poblacional se ha construido el panel $\mathrm{C}$ de la figura 1 con los datos de un estudio transversal de los determinantes de la cotinina en la población general (fumadores y no fumadores) ${ }^{14}$. La figura muestra la distribución bimodal de la concentración de cotinina en saliva y los posibles efectos (escenario ficticio) en esta distribución en el marco de una estrategia poblacional consistente, por ejemplo, en un aumento de los impuestos sobre los cigarrillos, la creación de espacios libres de humo o la mejora de la información al consumidor sobre los efectos del tabaco para la salud. El resultado esperado de esta intervención poblacional sería la reducción del número de fumadores y del consumo de cigarrillos, independientemente del nivel de riesgo individual de los fumadores, además de una importante reducción de la exposición al HAT en la población no fumadora.

De las medidas descritas por el Banco Mundial, el aumento de los impuestos sobre los cigarrillos y otros productos del tabaco y la creación de espacios libres de humo de tabaco en lugares públicos y centros de trabajo serían las principales estrategias que podriamos considerar poblaciones. El resultado esperado y deseable de estas intervenciones en la distribución poblacional del factor de riesgo sería, por un lado, cierto aplastamiento de la curva de fumadores debido al abandono del consumo por algunos fumadores, con el correspondiente incremento de la curva de no fumadores debido al aumento de exfumadores, y, por otro lado, un desplazamiento de la distribución poblacional (fumadores y no fumadores) de la concentración de cotinina hacia la izquierda, debido al menor consumo de tabaco y de exposición al HAT de los no fumadores (figura 1 panel $\mathrm{C}$ ). Este cambio de la distribución poblacional de los niveles de cotinina se traduce en una ganancia en salud en términos de reducción de la morbilidad y mortalidad de las enfermedades asociadas al consumo de tabaco como el cáncer de pulmón y el infarto de miocardio en las personas fumadoras y no fumadoras.

\section{Discusión}

La estrategia de "alto riesgo" en la prevención del tabaquismo centraría las intervenciones en la población fumadora independientemente del número de cigarrillos fumados y 
los niveles de cotinina en saliva. Estas personas son las que tienen mayor riesgo de desarrollar una enfermedad asociada al consumo de tabaco a corto y largo plazo ${ }^{25,26}$. En términos relativos, las intervenciones de "alto riesgo" estarían dirigidas al total de fumadores (diarios y ocasionales) de la población. Es decir, al 29,9\% de la población general mayor de 15 años expuesta a este factor de riesgo (fumadores y no fumadores) según los datos de la última encuesta de salud realizada en España el año 200927. Esta estrategia no solucionaría el verdadero problema de salud pública, debido a que excluimos de la intervención sanitaria a un gran número de individuos que, si bien no tienen un riesgo tan elevado, no están exentas de exposición y de cierto riesgo. En nuestro ejemplo de la epidemia del tabaquismo, dejariamos fuera de la intervención sanitaria al $70,1 \%$ de la población adulta ( $\geq 16$ años). Además, debido al alto porcentaje de población que está expuesta a bajos niveles de riesgo (personas no fumadoras con bajos niveles de cotinina en saliva), una parte de la casuística de la morbi-mortalidad atribuible al tabaco se atribuiría a las personas con menor exposición y riesgo. Esto ilustra el concepto de la "paradoja del riesgo" ya descrito por Rose: un gran número de personas expuestas a riesgos pequeños puede generar más casos que pocas personas expuestas a riesgos muy elevados ${ }^{1}$, como se desprendería de un análisis de las fracciones atribuibles poblacionales de carga de enfermedad asociada al tabaquismo pasivo ${ }^{11,28}$.

Otro punto de controversia en relación a la estrategia individual, especialmente en factores de riesgo como el tabaquismo, en el que existe un efecto dosis-respuesta y en el que ningún nivel de exposición se considera libre de riesgo $0^{6,7}$, es dónde fijar el límite o punto de corte para identificar a las personas de "alto riesgo", dejando por debajo de ese punto de corte una parte de la enfermedad causada por el factor de riesgo ${ }^{3}$. En nuestro ejemplo hemos planteado como punto de corte para deferencias la población de "alto riesgo" el consumo de tabaco, sin embargo, se podría definir niveles de cotinina superiores con mayor riesgo individual que el punto de corte que discrimina entre fumador y no fumador. Por otro lado, un aspecto positivo de la estrategia de "alto riesgo" es que las personas a las que está dirigida la intervención suelen estar más motivadas y tienen una mayor predisposición para eliminar el factor de riesgo. Sin embargo, en el caso del tabaquismo, los fumadores con mayores concentraciones de cotinina y más dependientes al consumo de tabaco en población general no son necesariamente los más motivados para dejar de fumar ${ }^{29}$. Además, la efectividad de esta estrategia individual podría verse afectada por las desigualdades sociales debido a que las clases sociales más desfavorecidas muestran más dificultad para dejar de fumar ${ }^{30}$. Aunque existen evidencias de desigualdades de género y clase social en la prevalencia de tabaquismo y la tasa de abandono a nivel poblacional ${ }^{31-33}$ una estrategia poblacional de este problema de salud no tendría por qué generar más desigualdades sociales $^{34,35}$.

Otra posible aproximación al riesgo individual de la población que consume tabaco de forma diaria, independientemente de la existencia de una enfermedad asociada, sería mediante la evaluación del grado de dependencia a la nicotina del fumador, medida por ejemplo a través del test de Fagerström ${ }^{36}$ que es un instrumento ampliamente utilizado en el ámbito clínico y que se asocia positivamente con la concentración de cotinina ${ }^{37}$. A partir del test de Fagerström se puede clasificar a la población fumadora con dependencia alta (puntuación mayor o igual a 7), dependencia media (puntuación entre 5 y 6) o dependencia baja (puntuación menor o igual a 4$)^{38}$.

Para ilustrar la estrategia de "alto riesgo" a partir del test de Fagerström se ha construido la figura 2 con datos de fumadores diarios provenientes del estudio transversal sobre los determinantes de la concentración de cotinina en la población de Barcelona ya mencionado ${ }^{12}$. La figura muestra la distribución de los fumadores diarios según su dependencia a la nicotina de acuerdo a la puntuación del test de Fagerström (barras) y la concentración media de cotinina en saliva (línea horizontal) y la concentración de cada sujeto (puntos) en cada una de las categorias de la puntuación del test. Basándonos en esta información, la población de alto riesgo correspondería a los fumadores con una alta dependencia, es decir, con una puntuación en el test de Fagerström mayor o igual a 7 puntos (11,2\% de los fumadores). Estas personas, además de tener una mayor dependencia a la nicotina, son las que tienen mayores niveles de cotinina en saliva (media de $307 \mathrm{ng} / \mathrm{ml}$ ).

Actualmente, existe cierta controversia sobre la hipótesis del "hardening" o "endurecimiento" de los fumadores ${ }^{39}$. Esta hipótesis sostiene que a medida que aumenta el número de exfumadores en la población, también aumenta el número de "grandes fumadores" ("hardcore" o "recalcitrant smokers" en inglés) que son los más dependientes a la nicotina y a los que más le cuesta abandonar el consumo ${ }^{40,41}$. Una posible implicación de la hipótesis del "hardening" es que las futuras intervenciones en el control del tabaquismo deberian ir dirigidas especificamente a este grupo poblacional de "alto riesgo". Pero esta visión de "alto riesgo" del problema de salud, como hemos visto de acuerdo con los postulados de Geoffrey Rose, no resolvería por completo el problema de salud ${ }^{9}$. Esto se debe a que los "grandes fumadores" son una parte del total de los fumadores que junto con los no fumadores constituirían la población diana de la estrategia poblacional. Además, si se aplicara de manera integral las seis medidas de control de tabaquismo propuestas por el Banco Mundial23 como una estrategia poblacional se esperaría que a medio o largo plazo la distribución poblacional de la concentración de cotinina en saliva (fumadores y no fumadores) se desplazara hacia la izquierda, tal y como se ilustra en la figura 1 (panel C). Los resultados de un estudio ecológico ${ }^{42}$ realizado en 27 países de la Unión Europea apoyan la estrategia poblacional, ya que muestra cómo la prevalencia de consumo de tabaco y de la exposición pasiva al HAT en casa y en el trabajo disminuye en los países con un mayor desarrollo e implementación de políticas de control de tabaquismo. Sin embargo, estas medidas poblacionales tienen un menor impacto en términos de tasas de abandono y de reducción del consumo que las estrategias de "alto riesgo", debido a la sensibilización por parte de la población diana. Este comportamiento es lo que Rose denominó "paradoja de la prevención": una 
medida preventiva que proporciona grandes beneficios para la comunidad le ofrece poco a cada uno de los individuos que participan en ella'.

\section{Conclusión}

La estrategia poblacional propuesta por Geoffrey Rose para los factores de riesgo como la hipertensión arterial o la hipercolesterolemia también muestra su aplicabilidad en el control de la epidemia del tabaquismo. En estos momentos en que se han planteado políticas públicas de control de tabaquismo en todo el mundo parece conveniente tener en consideración la utilidad de la estrategia poblacional para el abordaje de este importante problema de salud, sin menospreciar intervenciones seleccionadas también de probada eficacia dirigidas a colectivos de "alto riesgo".

\section{Financiación}

Este estudio ha sido financiado por el Instituto de Salud Carlos III (RTICC RD06/0020/0089); el Departamento de Universidades e Investigación de la Generalitat de Catalunya (SGR200500646); y la V Ayuda "Enrique Nájera" para jóvenes epidemiólogos de la Sociedad Española de Epidemiología, financiada por la Escuela Nacional de Sanidad.

\section{Conflictos de intereses}

Los autores declaran no tener conflictos de intereses.

\section{Referencias}

1. Rose G. La estrategia de la medicina preventiva. Barcelona: Masson-Salvat Medicina; 1994.

2. Rose G. Sick individuals and sick populations. Int J Epidemiol 2001; 30: 427-32.

3. Porta M. A Dictionary of Epidemiology (Fifth Edition). Nueva York: Oxford University Press; 2008.

4. Doyle YG, Furey A, Flowers J. Sick individuals and sick populations: 20 years later. J Epidemiol Community Health 2006; 60: 396-8

5. Charlton BG. A critique of Geoffrey Rose's 'population strategy' for preventive medicine. J R Soc Med 1995; 88: 607-10.

6. US Department of Health and Human Services. The health consequences of smoking. Atlanta, GA: US Department of Health and Human Services, Centers for Disease Control and Prevention, National Center for Chronic Disease Prevention and Health Promotion, Office on Smoking and Health; 2004.

7. US Department of Health and Human Services. The health consequences of involuntary exposure to tobacco smoke: a report of the Surgeon General. Atlanta, GA: US Department of Health and Human Services, Centers for Disease Control and Prevention, National Center for Chronic Disease Prevention and Health Promotion, Office on Smoking and Health; 2006.
8. World health Organization (WHO). MPOWER. WHO Report on the Global tobacco epidemic. Genève: WHO; 2008.

9. Chaiton MO, Cohen JE, Frank J. Population health and the hardcore smoker: Geoffrey Rose revisited. J Public Health Policy 2008; 29: 307-18.

10. Banegas JR, Díez-Gañán $L$, Bañuelos-Marco B, González EJ, Villar $A F$, Martín-Moreno JM, et al. Mortalidad atribuible al consumo de tabaco en España en 2006. Med Clin 2011; 136: 97-102.

11. López MJ, Pérez-Ríos M, Schiaffino A, Nebot M, Montes A, Ariza C, et al. Mortality attributable to passive smoking in Spain, 2002. Tob Control 2007; 16: 373-7.

12. Fu M, Fernández $E$, Martínez-Sánchez JM, Pascual JA, Schiaffino A, Agudo A, et al. Salivary cotinine concentrations in daily smokers in Barcelona, Spain: a cross-sectional study. BMC Public Health 2009; 9: 320.

13. Haw SJ, Gruer L. Changes in exposure of adult non-smokers to secondhand smoke after implementation of smoke-free legislation in Scotland: national cross sectional survey. BMJ 2007; 335: 549-52.

14. Martínez-Sánchez JM, Fernández E, Fu M, Pascual JA, Ariza C, Agudo $A$, et al. Assessment of exposure to secondhand smoke by questionnaire and salivary cotinine in the general population of Barcelona, Spain (2004-2005). Prev Med 2009; 48: 218-23.

15. Allwright S, Paul G, Greiner B, Mullally BJ, Pursell L, Kelly A, et al. Legislation for smoke-free workplaces and health of bar workers in Ireland: before and after study. BMJ 2005; 331: 1117.

16. Semple S, Maccalman L, Naji AA, Dempsey S, Hilton S, Miller BG, et al. Bar workers' exposure to second-hand smoke: the effect of Scottish smoke-free legislation on occupational exposure. Ann Occup Hyg 2007; 51: 571-80.

17. Fernández $E, F u$ M, Pascual JA, López MJ, Pérez-Ríos M, Schiaffino $A$, et al. Impact of the Spanish smoking law on exposure to second-hand smoke and respiratory health in hospitality workers: a cohort study. PLoS One 2009; 4: e4244.

18. Jarvis MJ, Fidler J, Mindell J, Feyerabend C, West R. Assessing smoking status in children, adolescents and adults: cotinine cut-points revisited. Addiction 2008; 103: 1553-61.

19. Benowitz NL, Bernert JT, Caraballo RS, Holiday DB, Wang J. Optimal serum cotinine levels for distinguishing cigarette smokers and nonsmokers within different racial/ethnic groups in the United States between 1999 and 2004. Am J Epidemiol 2009; 169: 236-48.

20. Martínez-Sánchez JM, Fu M, Ariza C, López MJ, Saltó E, Pascual $J A$, et al. Punto de corte óptimo de la concentración de cotinina en saliva para discriminar entre fumadores y no fumadores en la población adulta de Barcelona. Gac Sanit 2009; 23: 501-5.

21. World Health Organization (WHO). Convenio Marco de la OMS para el Control del Tabaco. Ginebra: WHO; 2003.

22. World Health Organization (WHO). Parties to the WHO Framework Convention on Tobacco Control. Disponible en: http://www.who.int/fctc/signatories_parties/en/index.html [Consultado el 20 de Mayo de 2011]

23. World Bank. Tobacco control at a glance. Washington DC: World Bank; 2003. Disponible en: www.worldbank.org/tobacco [Consultado el 20 de Mayo de 2011]

24. Levy DT, Chaloupka F, Gitchell J. The effects of tobacco control policies on smoking rates: a tobacco control scorecard. J Public Health Manag Pract 2004; 10: 338-53. 
25. Boffetta P, Clark S, Shen M, Gislefoss R, Peto R, Andersen A. Serum cotinine level as predictor of lung cancer risk. Cancer Epidemiol Biomarkers Prev 2006; 15: 1184-8.

26. Al-Delaimy WK, Willett WC. Toenail nicotine level as a novel biomarker for lung cancer risk. Am J Epidemiol 2011; 173: 8228.

27. Instituto Nacional de Estadistica. Encuesta Europea de Salud. Año 2009. Disponible en: http://www.ine.es/inebmenu/mnu_ salud.htm. [Consultado el 20 de mayo de 2011].

28. Öberg M, Jaakkola MS, Woodward A, Peruga A, Pruss-Ustun A. Worldwide burden of disease from exposure to second-hand smoke: a retrospective analysis of data from 192 countries. Lancet 2011; 377: 139-46.

29. Fu M, Fernández E, Pascual JA, Martínez-Sánchez JM, Agudo $A$, Moncada $A$, et al. Stages of change, smoking characteristics, and cotinine concentrations in smokers: Setting priorities for smoking cessation. Prev Med 2011; 52: 139-145.

30. Fernández $E_{1}$ Schiaffino $A$, Borrell $C$, Benach J, Ariza $C$, Ramón $J M$, et al. Social class, education, and smoking cessation: Longterm follow-up of patients treated at a smoking cessation unit. Nicotine Tob Res 2006; 8: 29-36.

31. Regidor E, Gutiérrez-Fisac JL, Calle ME, Navarro P, Domínguez $V$. Trends in cigarette smoking in Spain by social class. Prev Med 2001; 33: 241-8.

32. Fernández $E$, Schiaffino $A$, García $M$, Borràs JM. Widening social inequalities in smoking cessation in Spain, 1987-1997. J Epidemiol Community Health 2001; 55: 729-30.

33. Schiaffino $A$, Fernández $E$, Kunst $A$, Borrell $C$, Garcia $M$, Borràs $J M$, et al. Time trends and educational differences in the incidence of quitting smoking in Spain (1965-2000). Prev Med 2007; 45: 226-32.

34. Main C, Thomas S, Ogilvie D, Stirk L, Petticrew M, Whitehead M, et al. Population tobacco control interventions and their effects on social inequalities in smoking: placing an equity lens on existing systematic reviews. BMC Public Health 2008; 8: 178.

35. Thomas S, Fayter D, Misso K, Petticrew M, Sowden A, Whitehead $M$ et al. Population tobacco control interventions in smoking: systematic review. Tob Control 2008; 17: 230-237.

36. Heatherton TF, Kozlowski LT, Frecker RC, Fagerström KO. The Fagerström Test for Nicotine Dependence: a revision of the Fagerström Tolerance Questionnaire. Br J Addict 1991; 86: 111927.

37. Fu M, Martínez-Sánchez JM, Agudo A, Pascual JA, Ariza C, Moncada $A$, et al. (en prensa) Nicotine dependence and salivary cotinine concentrations in daily smokers: a population-based cross-sectional study in Spain. Eur J Cancer Prev.

38. Barrueco Ferrero M, Hernández Mezquita MA, Torrecillas Garcia M. Manual de prevención y tratamiento del tabaquismo (3 Edición). Majadahonda (Madrid): ERGON; 2006.

39. Warner KE, Burns DM. Hardening and the hard-core smoker: concepts, evidence, and implications. Nicotine Tob Res 2003; 5: 37-48.

40. Fagerström K, Furberg H. A comparison of the Fagerström Test for Nicotine Dependence and smoking prevalence across countries. Addiction 2008; 103: 841-5.

41. Fu M, Martínez-Sánchez JM, Pérez-Ríos M, López MJ, Fernández E. A comparison of the Fagerström test for nicotine dependence and smoking prevalence across countries: updated data from Spain. Addiction 2009; 104: 326-7.
42. Martínez-Sánchez JM, Fernández E, Fu M, Gallus S, Martínez $C$, Sureda $X$, et al. Smoking behaviour, involuntary smoking, attitudes towards smoke-free legislation, and tobacco control activities in the European Union. PLoS One 2010; 5(11): e13881. 
